\title{
Identification of DNA methylation-driven genes by integrative analysis of DNA methylation and transcriptome data in pancreatic adenocarcinoma
}

\author{
WEI ZHANG ${ }^{1}$, SHUAI SHANG ${ }^{1}$, YINGYING YANG ${ }^{1}$, PEIYAO LU ${ }^{1}$, \\ TENG WANG $^{1}$, XINYI CUI $^{1}$ and XUEXI TANG ${ }^{1,2}$ \\ ${ }^{1}$ Department of Marine Ecology, College of Marine Life Sciences, Ocean University of China, Qingdao, Shandong 266003; \\ ${ }^{2}$ Laboratory for Marine Ecology and Environmental Science, Qingdao National Laboratory of Oceanology for \\ Marine Science and Technology, Qingdao, Shandong 266071, P.R. China
}

Received July 24, 2019; Accepted January 10, 2020

DOI: $10.3892 /$ etm.2020.8554

\begin{abstract}
Pancreatic adenocarcinoma (PAAD) is a painful and fatal disease that undoubtedly remains a health care priority and offers significant therapeutic challenges. The significance of epigenetic modifications, including DNA methylation in tumor development, has gained the attention of researchers. Identifying DNA methylation-driven genes and investigating the mechanisms underlying the tumorigenesis of PAAD are of substantial importance for developing methods of physiological evaluation, treatment planning and prognostic prediction for PAAD. In the present study, a comprehensive analysis of DNA methylation and gene expression data from 188 clinical samples was performed to identify DNA methylation-driven genes in PAAD. In addition, the diagnostic and prognostic value of DNA methylation-driven genes was evaluated using receiver operating characteristic curve, survival and recurrence analyses. A total of 7 DNA methylation-driven genes, namely zinc finger protein 208 (ZNF208), eomesodermin (EOMES), prostaglandin D2 receptor (PTGDR), chromosome 12 open reading frame 42 (C12orf42), integrin subunit $\alpha 4$ (ITGA4), dedicator of cytokinesis 8 and protein phosphatase 1 regulatory inhibitor subunit 14D (PPP1R14D), were identified. All of them may be used to diagnose PAAD with excellent specificity and sensitivity (area under curve, $>0.8$ ). Of the 7 DNA methylation-driven genes, 6 were significantly associated with overall survival (OS) and
\end{abstract}

Correspondence to: Professor Xuexi Tang, Department of Marine Ecology, College of Marine Life Sciences, Ocean University of China, 5 Yushan Road, Qingdao, Shandong 266003, P.R. China E-mail: zw@stu.ouc.edu.cn

Abbreviations: PAAD, pancreatic adenocarcinoma; ROC, receiver operating characteristic; TCGA, The Cancer Genome Atlas; AUC, area under the curve

Key words: pancreatic adenocarcinoma, DNA methylation-driven gene, diagnosis, prognosis, recurrence recurrence-free survival (RFS) $\mathrm{P}<0.05)$. Among them, ZNF208, EOMES, PTGDR, C12orf42 and ITGA4 were significantly negatively associated with the OS rate and positively associated with the recurrence rate, while PPP1R14D was significantly positively associated with the OS rate and negatively associated with the recurrence rate. The present study provides novel insight into the epigenetic alterations associated with the occurrence and progression of PAAD, thereby increasing the mechanistic understanding of this disease, offering potential novel molecular biomarkers and contributing to the development of therapeutic targets for PAAD.

\section{Introduction}

Pancreatic cancer is frequently fatal, with a generally poor prognosis (1). Pancreatic adenocarcinoma (PAAD) is the most common subtype of pancreatic cancer in humans and is responsible for $>85 \%$ of pancreatic cancer cases (2). It is projected to become the second leading cause of cancer-associated death in Western societies within a decade (3). Its dismal prognosis is due to rapid disease progression and early metastasis, leading to late diagnosis and a high level of resistance to treatment (4). Growing evidence has revealed a relatively complex underlying mechanism of the disease in terms of development and progression, including the interaction between epigenomic and genomic alterations (5). Despite the understanding of aberrant gene networks gained from previous studies, the epigenomic interactions in PAAD remain to be fully elucidated (6).

At present, the genetic and phenotypic changes associated with the pathogenesis of pancreatic cancer are increasingly attracting attention (7). As an epigenetic modification, DNA methylation is critical for maintaining genomic and chromosomal structural stability, gene imprinting and gene silencing (8-10). DNA methylation appears primarily on cytosine residues in $\mathrm{CpG}$ sites, which exist throughout the genome. Areas with abundant $\mathrm{CpG}$ sites are called $\mathrm{CpG}$ islands. Altered DNA methylation is linked to the initiation and progression of various types of cancer, and high levels of methylation in $\mathrm{CpG}$ islands and promoter regions may lead to transcriptional silencing of tumor suppressor genes (8). 
By contrast, hypomethylation may lead to overexpression of oncogenes and genomic instability.

High-throughput methods have been used to assess the epigenetic changes associated with the development and progression of PAAD (11). Studies have indicated that a variety of genes in pancreatic cancer, including Ras association domain family 1 isoform A (RASSF1A), p16, suppressor of cytokine signalling 1 (SOCS-1) and neuronal pentraxin 2 (NPTX2), are abnormally methylated and are critical for the pathogenesis of pancreatic cancer (12-14). However, a large number of statistically significant methylation events identified by high-throughput screening have no association with gene expression changes. A high-throughput method is required to integrate data across multiple platforms to determine the epigenetic events that are most likely to be associated with PAAD.

The Cancer Genome Atlas (TCGA) indicates a significant diversity of genetic and epigenetic variations in PAAD (15). Numerous platforms used in the TCGA database enable the analysis of data integrated from multiple sources to identify specific abnormalities that are most likely to result in a carcinogenic process. However, no integrated DNA methylation and transcriptome data are available and it is currently not possible to identify DNA methylation-driven genes in PAAD. MethylMix is a method of applying three criteria to identify methylation-driven genes in diseases by integrating DNA methylation and transcriptome data (16). First, the determination of the degree of methylation should not depend on any threshold that is normally used. Furthermore, the hypermethylation or hypomethylation of a gene in cancer must be evaluated in comparison with normal tissue. Finally, the identification of highly methylated or hypomethylated genes should be selective for those with predicted effects on transcription, meaning that their methylation is functionally associated.

Despite the improvement in the ability to diagnose PAAD over time, it remains difficult to differentiate malignant tumors from benign disease. Endoscopic ultrasound-fine-needle aspiration provides a tissue sample for cytological studies, thereby helping resolve this issue; however, an improper biopsy reduces the diagnostic value (17). Adjuvant tests may be performed to screen for biomarkers in tissue samples to reduce the requirement for biopsy. DNA methylation is currently being assessed as a biomarker. Epigenetic changes do not lead to DNA sequence mutations, but they serve as causal links between genes and phenotypes. DNA methylation is one epigenetic mechanism (18). Epigenetic changes may activate or suppress numerous signaling pathways, thereby causing cancer. Epigenetic changes may occur in the early development stage of tumors; thus, they may serve as biomarkers that may be able to help identify and prevent cancer in the early stage (19). As PAAD-specific biomarkers with high diagnostic value are not sufficient, the identification of PAAD-specific DNA methylation markers with high specificity and sensitivity will help further enhance the ability of clinicians to diagnose PAAD.

PAAD is a painful and potentially fatal disease that is an important health concern and poses numerous therapeutic challenges. The significance of DNA methylation in the development of PAAD is gaining attention. However, to date, no comprehensive analysis of DNA methylation and transcriptome data has been performed to identify DNA methylation-driven genes in PAAD. Therefore, identifying DNA methylation-driven genes and investigating the mechanisms underlying the tumorigenesis of PAAD are of substantial importance to treatment planning and prognostic prediction for PAAD. The present study reports on the analysis of large-scale methylation data using Infinium $450 \mathrm{k}$ methylation arrays (Illumina) and expression profiles using RNA sequencing. A total of 7 DNA methylation-driven genes were identified, all of which may serve as important markers for the diagnosis of PAAD. Furthermore, 6 of them were associated with cancer recurrence and patient survival. These DNA methylation-driven genes are critical for elucidating PAAD progression and may serve as future therapeutic biomarkers.

\section{Materials and methods}

Screening for differentially expressed genes (DEGs). In total, 188 DNA methylation profiles (178 PAAD samples together with 10 non-tumor samples), 182 RNA-sequencing (RNA-seq) profiles (178 PAAD samples together with 4 non-tumor samples), and the associated clinical PAAD data were downloaded from the TCGA database (https://portal.gdc. cancer.gov/) (Table SI). Of the 178 patients with PAAD, 178 patients had overall survival (OS) data and 155 patients had recurrence-free survival (RFS) data. To screen for the DEGs between normal pancreatic tissue and PAAD, the 'edgeR' package in $\mathrm{R}$ was used (20) England. A false discovery rate $(\mathrm{FDR})<0.05$ and $\mathrm{a} \mid \log _{2}$ fold change $(\mathrm{FC}) \mid>2$ were defined as the cut-off criteria to select genes for further analysis.

Integrated analysis of DNA methylation and gene expression. Level 3 DNA methylation data and clinical information, including time of death, follow-up time and status of PAAD, were downloaded from TCGA database on August 1, 2018. An analysis combining gene expression (RNA-seq) data and methylation data was performed using the 'MethylMix' package in $\mathrm{R}$ to identify DNA methylation-driven genes (16). MethylMix is a program designed to identify methylation events associated with gene expression (16). MethylMix requires three datasets: Disease DNA methylation data, matched disease gene expression data and normal DNA methylation data. The MethylMix analysis has three parts: First, disease DNA methylation data are combined with the matched disease gene expression data to identify the methylation events leading to changes in gene expression, and only genes that pass the correlation filter are selected for further analysis; second, a beta mixed model is used to define the methylation state in a large number of patients, eliminating the need for any threshold; and third, the Wilcoxon rank sum test is used to compare the DNA methylation statuses between normal samples and cancer samples. Multiple testing is calculated using a q cutoff of 0.05 . The final result is a differential methylation (DM) value, where a positive DM value represents a high degree of methylation and a negative DM value represents a low degree of methylation.

Receiver operating characteristic (ROC) analyses. For the assessment of the diagnostic values of DNA methylation-driven genes in PAAD, ROC analyses were performed using the 'pROC' package in R. The ROC curve was generated and the area under the curve (AUC) with the binomial exact confidence interval was calculated. For AUC values $>0.7$, the hub gene 

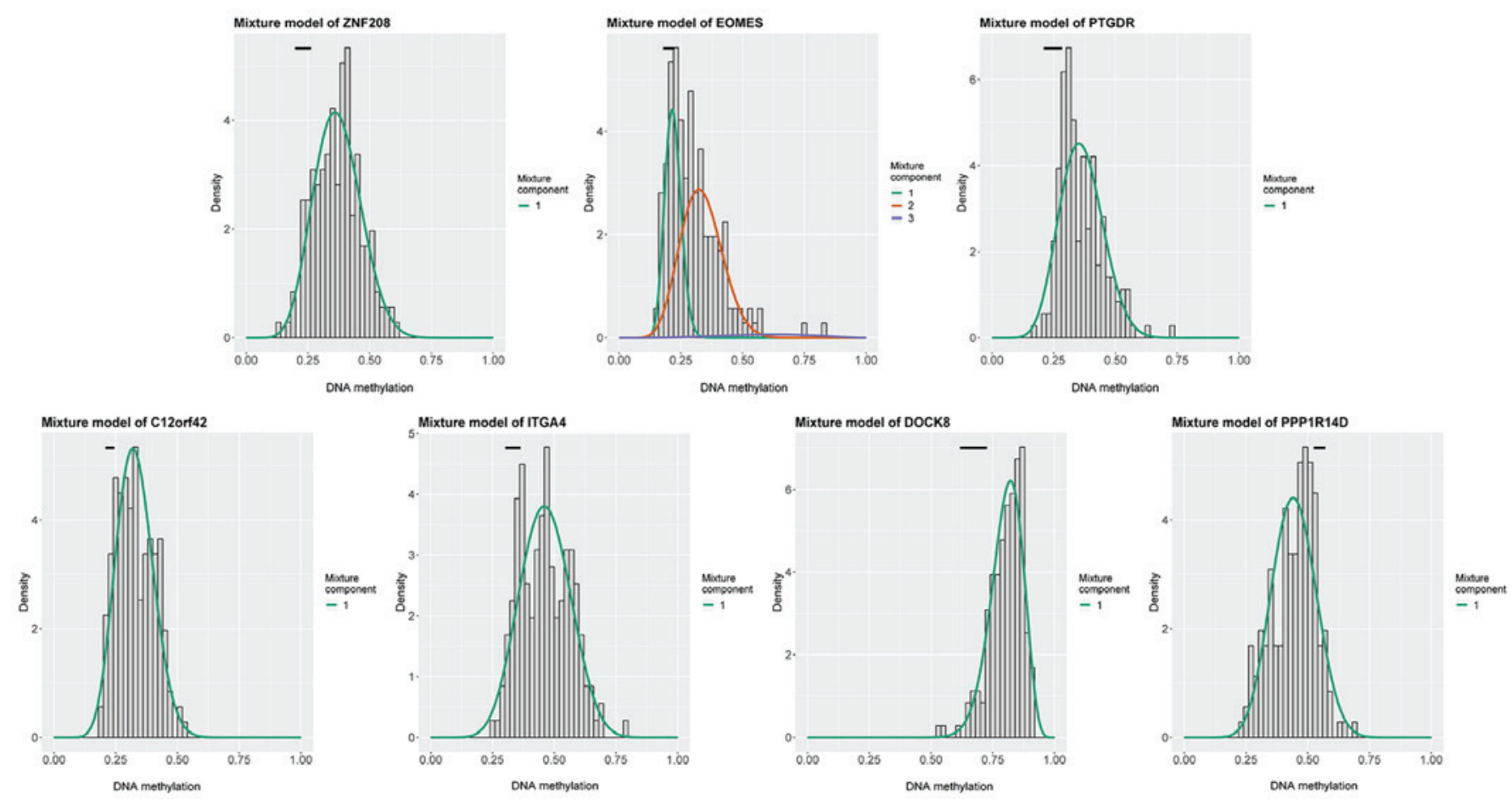

Figure 1. Mixed models obtained from the 'MethylMix' R package for the 7 DNA methylation-driven genes. The histogram demonstrates the distribution of methylation in tumor samples (indicated by beta values where higher beta values denote higher levels of methylation). The horizontal black bar indicates the distribution of methylation values in the normal samples. The curve indicates the simulated trend curve of the methylation distribution of tumor samples. The fewer the curves, the more stable the model is. The black horizontal line indicates the methylation level distribution of the normal samples. ZNF208, zinc finger protein 208; EOMES, eomesodermin; PTGDR, prostaglandin D2 receptor; C12orf42, chromosome 12 open reading frame 42; ITGA4, integrin subunit $\alpha$ 4; DOCK8, dedicator of cytokinesis 8; PPP1R14D, protein phosphatase 1 regulatory inhibitor subunit 14D.

was deemed able to distinguish between normal pancreatic tissue and PAAD with excellent specificity and sensitivity.

Survival analysis. For the survival analysis, 178 patients with DNA methylation data and OS data were dichotomized into two groups (high vs. low) based on the optimal cutoff value for each DNA methylation-driven gene. Subsequently, the 'survival' package in $\mathrm{R}$ was adopted to generate the Kaplan-Meier survival curve and perform the log-rank test. Multivariate Cox regression analyses were then performed to investigate whether the prognostic value of the gene was independent of TNM stage.

Recurrence analysis. The correlation between the 7 DNA methylation-driven genes and recurrence may provide a preliminary indication of the role of these genes in the prognostic prediction and the aspects of PAAD involved. In the recurrence analysis, 155 patients with DNA methylation data and RFS data were dichotomized into two groups (high vs. low) based on the optimal cutoff value for each DNA methylation-driven gene. Recurrence analysis was performed as described above.

Gene set enrichment analysis (GSEA). For the GSEA, 178 PAAD samples with DNA methylation data were divided into two groups (high vs. low) according to the DNA methylation level of each DNA methylation-driven gene and the median DNA methylation value was used as the cut-off point. To gain insight into the function of each DNA methylation-driven gene, GSEA (version 3.0; http://software.broadinstitute. org/gsea/index.jsp) was performed with these two groups.
The annotated gene sets c2.cp.kegg.v5.2.symbols.gmt were selected as the reference gene sets. $\mathrm{P}<0.05$ was considered to indicate a statistically significant difference.

\section{Results}

DEGs in PAAD. The RNA expression levels in 178 PAAD tissues and 4 normal tissues were analyzed. Genes meeting the cut-off criteria of a corrected $\mathrm{P}<0.05$ and $\log _{2} \mathrm{FCl}>2$ were regarded as differentially expressed. Thereby, 814 (65.92\%) downregulated genes and 30 (34.08\%) upregulated genes were identified.

Identification of DNA methylation-driven genes. DNA methylation data for 10 normal tissues and 178 PAADs and gene expression data for the DEGs identified in the 178 PAADs were included in the analysis. Thresholds of a corrected P-value for differential expression between normal tissues and PAAD of $<0.05$ and a correlation between gene expression and DNA methylation of <- 0.3 were used. A total of 7 DNA methylation-driven genes, namely zinc finger protein 208 (ZNF208), eomesodermin (EOMES), prostaglandin D2 receptor (PTGDR), chromosome 12 open reading frame 42 (C12orf42), integrin subunit $\alpha 4$ (ITGA4), dedicator of cytokinesis 8 (DOCK8) and protein phosphatase 1 regulatory inhibitor subunit 14D (PPP1R14D), were identified (Table SII). Of these genes, ZNF208, EOMES, PTGDR, C12orf42, ITGA4 and DOCK8 were hypermethylated, while PPP1R14D was hypomethylated (Fig. 1; Table SII). Fig. 2 displays the inverse correlation between DNA methylation and the matched gene expression of the 7 methylation-driven genes. 


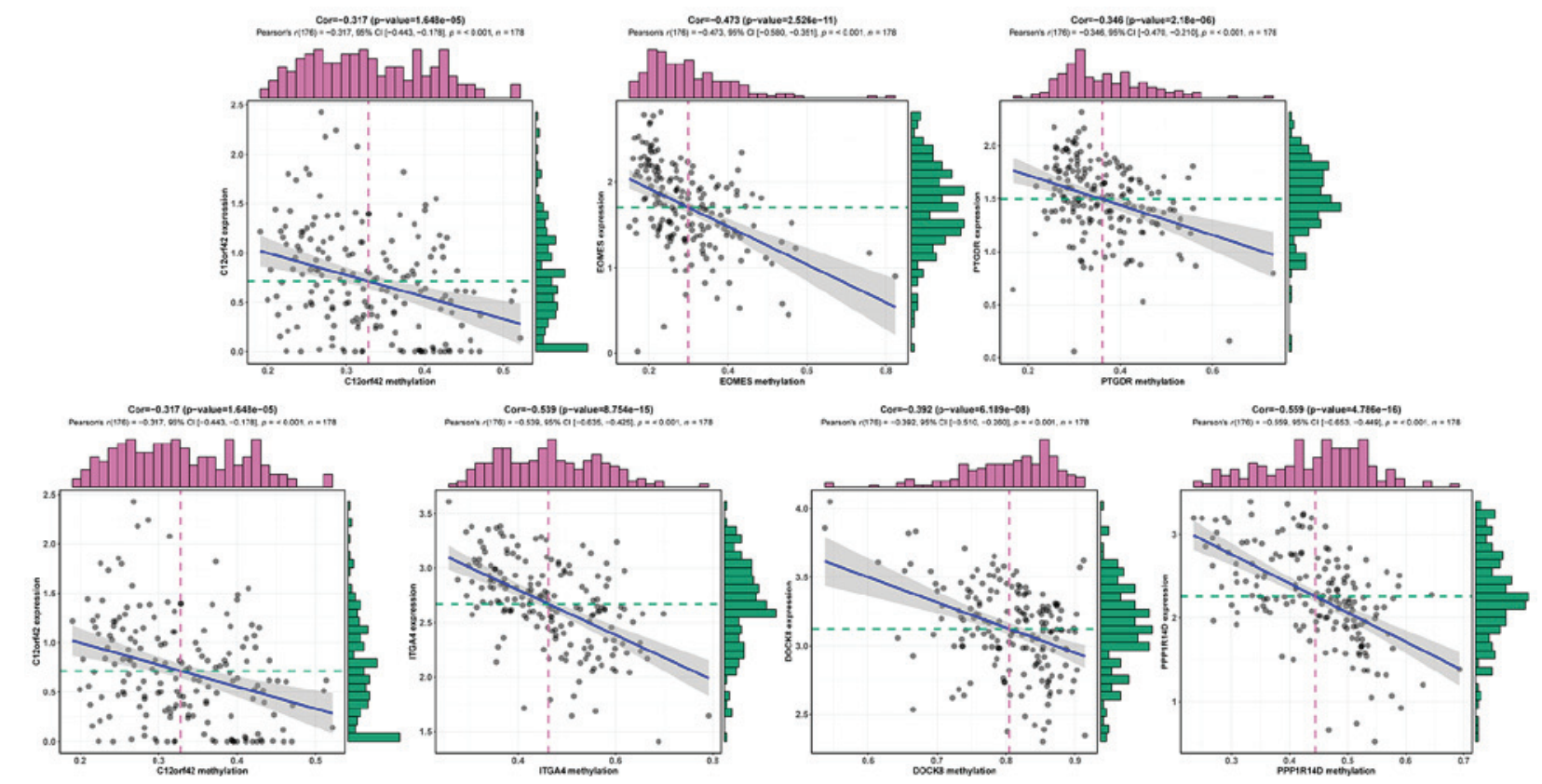

Figure 2. Regression analysis between the DNA methylation and mRNA expression of the 7 DNA methylation-driven genes. The horizontal axis and vertical axis represent DNA methylation-driven gene expression and DNA methylation, respectively. Histograms of gene expression and DNA methylation are provided at the upper and right edges. Cor, correlation coefficient; ZNF208, zinc finger protein 208; EOMES, eomesodermin; PTGDR, prostaglandin D2 receptor; C12orf42, chromosome 12 open reading frame 42; ITGA4, integrin subunit $\alpha 4$; DOCK8, dedicator of cytokinesis 8; PPP1R14D, protein phosphatase 1 regulatory inhibitor subunit $14 \mathrm{D}$.
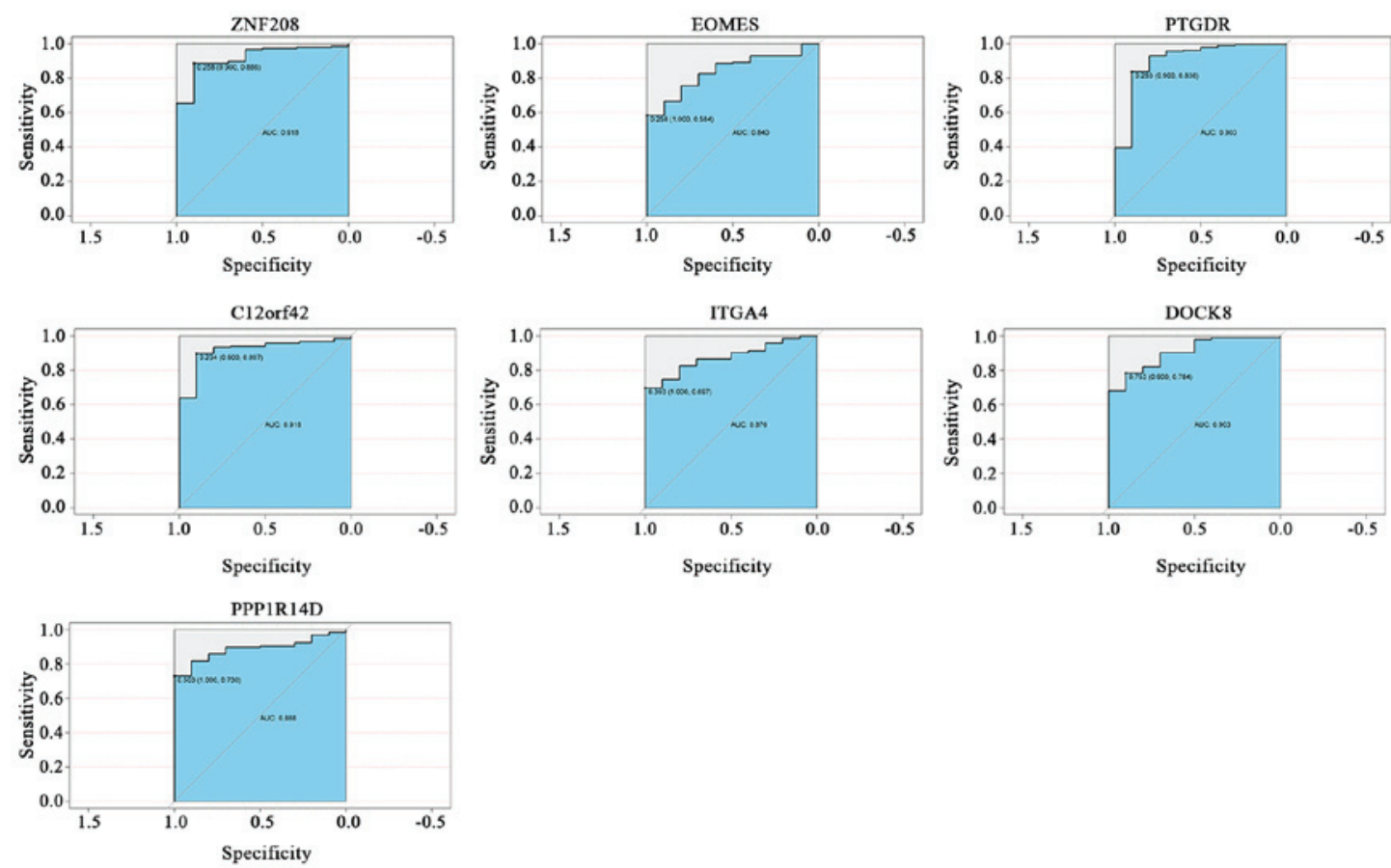

Figure 3. ROC curves of DNA methylation-driven genes for distinguishing between pancreatic adenocarcinoma and healthy controls. The cutoff value for determining the AUC was obtained from the 'roc' function of the 'pROC' R package. The left and right values in the brackets indicate the specificity and sensitivity of the cutoff value, respectively. The ROC curves indicate the diagnostic ability of the DNA methylation-driven genes, with the x-axis and y-axis displaying the specificity and sensitivity, respectively. ROC, receiver operating characteristic; AUC, area under curve; ZNF208, zinc finger protein 208; EOMES, eomesodermin; PTGDR, prostaglandin D2 receptor; C12orf42, chromosome 12 open reading frame 42; ITGA4, integrin subunit $\alpha$ 4; DOCK8, dedicator of cytokinesis 8; PPP1R14D, protein phosphatase 1 regulatory inhibitor subunit 14D.

Diagnostic efficiency of DNA methylation-driven genes. To assess the diagnostic ability of the 7 DNA methylation-driven genes in the TCGA dataset, ROC curve analyses were performed and calculate the AUCs. The AUCs of ZNF208
(0.918), EOMES (0.840), PTGDR (0.903), C12orf42 (0.918), ITGA4 (0.876), DOCK8 (0.903) and PPP1R14D (0.888) were all $>0.8$, indicating that the 7 DNA methylation-driven genes, particularly ZNF208, PTGDR, C12orf42 and DOCK8, 

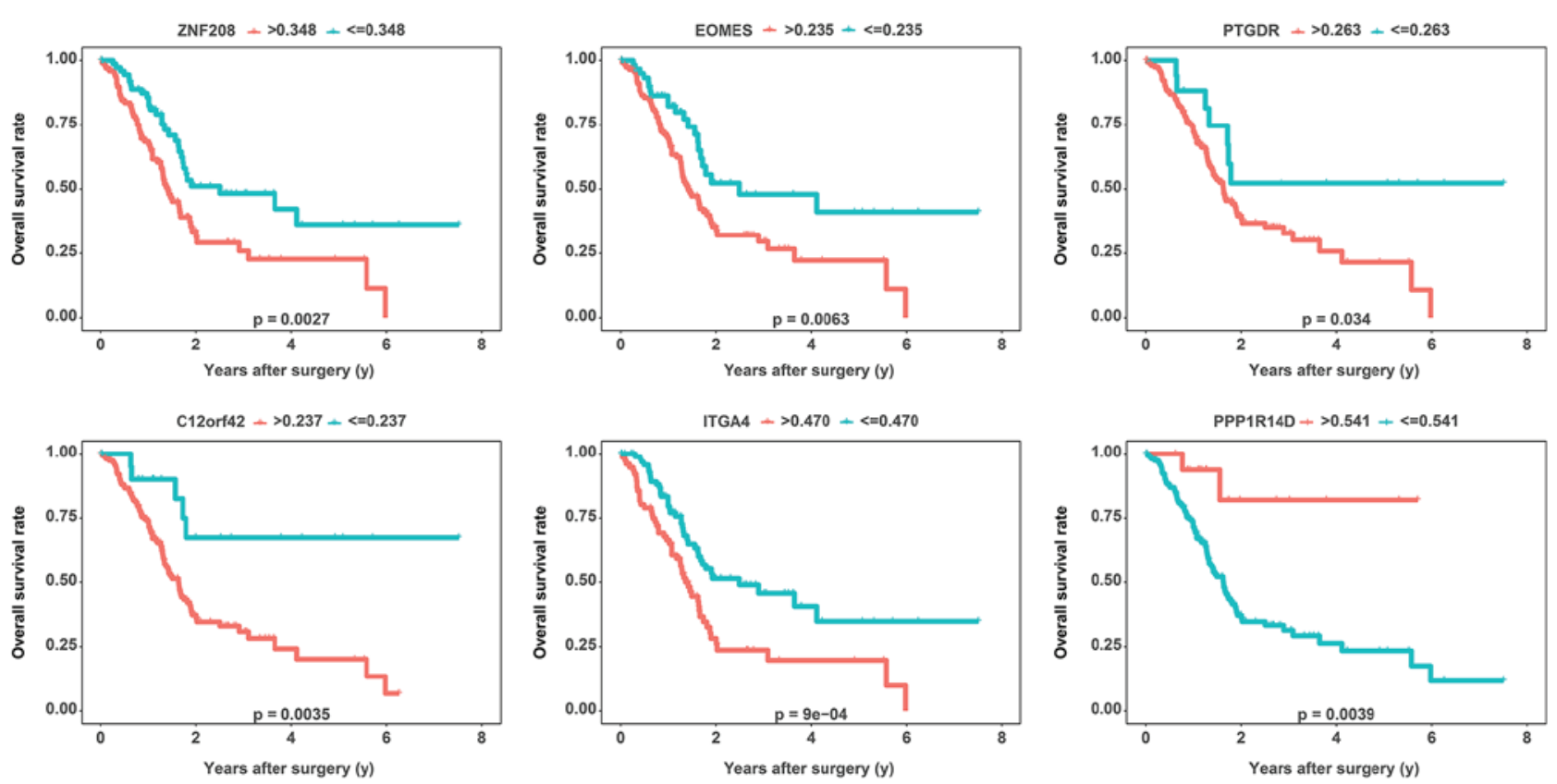

Figure 4. Cumulative survival rates associated with the 6 DNA methylation-driven genes in pancreatic adenocarcinoma. The cutoff value is displayed above the survival curve. The log-rank $\mathrm{P}$ is displayed below the survival curve. The $\mathrm{x}$-axis and $\mathrm{y}$-axis display the survival time (years) and cumulative overall survival rate, respectively. ZNF208, zinc finger protein 208; EOMES, eomesodermin; PTGDR, prostaglandin D2 receptor; C12orf42, chromosome 12 open reading frame 42; ITGA4, integrin subunit $\alpha$ 4; PPP1R14D, protein phosphatase 1 regulatory inhibitor subunit 14D.

exhibited excellent diagnostic efficiency for PAAD (Fig. 3). For the diagnosis of PAAD, the sensitivity and specificity of ZNF208 were 88.6 and $90.0 \%$, respectively; those of PTGDR were 83.8 and $90.0 \%$, respectively; those of C12orf42 were 89.7 and $90.0 \%$, respectively; and the sensitivity and specificity of DOCK8 were 78.4 and $90.0 \%$, respectively.

Survival prediction using the DNA methylation-driven genes. For the analysis of the potential usefulness of DNA methylation-driven genes for the prognostic prediction for PAAD, Kaplan-Meier survival curves were generated and log-rank tests were performed for each of the DNA methylation-driven genes. In total, 176 patients with clinical data, including OS and survival status, were divided into high and low DNA methylation status groups with respect to the 7 DNA methylation-driven genes according to their respective optimal cutoffs based on the 'surv_cutpoint' function of the 'survminer' $\mathrm{R}$ package. Finally, 5 DNA methylation-driven genes (ZNF208, EOMES, PTGDR, C12orf42 and ITGA4) were considered to be significantly negatively associated with the survival time of patients with PAAD $(\mathrm{P}<0.05$ by log-rank test), while PPP1R14D was considered to be significantly positively associated with the survival time of PAAD patients $(\mathrm{P}<0.01$ by log-rank test $)$ (Fig. 4; Table SIII). However, DOCK8 was not significantly associated with the OS of patients with PAAD (Table SIII). To determine whether these genes were independent of the TNM stage with regard to the prediction of the prognosis of patients with PAAD, a multivariate analysis was performed for each gene. Multivariate Cox regression analysis revealed that after adjusting for TNM stage, $\mathrm{C} 12$ orf42 $[\mathrm{P}=0.012$, hazard ratio (HR): 3.304, 95\% CI: 1.302-8.383], PPP1R14D ( $\mathrm{P}=0.021$, HR: 0.189, 95\% CI: 0.046-0.774), EOMES (P=0.024, HR: 1.744, 95\% CI: $1.075-2.830), \mathrm{ZNF} 208(\mathrm{P}=0.006, \mathrm{HR}: 1.885$, 95\% CI: 1.202-2.954) and ITGA4 (P=0.012, HR: 3.304, 95\%
CI: 1.302-8.383) were still significantly associated with OS, indicating that C12orf42, PPP1R14D, EOMES, ZNF208 and ZGA4 are independent of the TNM stage. However, as the number of patients was relatively small, PTGDR did not achieve statistical significance $(\mathrm{P}=0.083, \mathrm{HR}: 2.050,95 \% \mathrm{CI}$ : 0.910-4.617).

Recurrence prediction using DNA methylation-driven genes. To explore the association between the 7 identified DNA methylation-driven genes and the recurrence rate of PAAD, the cumulative recurrence rates associated with the 7 DNA methylation-driven genes in patients with PAAD were investigated using Kaplan-Meier curve analysis. In total, 154 patients with clinical data, including RFS and relapse status, were divided into high and low DNA methylation status groups with respect to the methylation status of each of the 7 DNA methylation-driven genes according to their respective optimal cutoffs based on the 'surv_cutpoint' function of the 'survminer' R package. Of the 7 DNA methylation-driven genes, 6 exhibited an obvious association with the recurrence rate based on their respective optimal cut-offs. A total of five DNA methylation-driven genes (ZNF208, EOMES, PTGDR, C12orf42 and ITGA4) were positively associated with the recurrence rate, while PPP1R14D was negatively associated with the recurrence rate (log-rank $\mathrm{P}<0.05$ ) (Fig. 5; Table SIV). However, DOCK8 did not have a significant correlation with the recurrence rate of PAAD (Table SIV). This result is consistent with the result of the OS analysis. To determine whether these genes were predictors of RFS independent of the TNM stage, a multivariate analysis was performed for each gene. The multivariate Cox regression analyses revealed that after adjusting for TNM stage, PTGDR $(\mathrm{P}=0.031, \mathrm{HR}$ : 4.966, 95\% CI: 1.159-21.271), EOMES ( $\mathrm{P}=0.002$, HR: 2.298 , 95\% CI: 1.346-3.922), ZNF208 ( $\mathrm{P}=0.045$, HR: 4.474, 95\% 

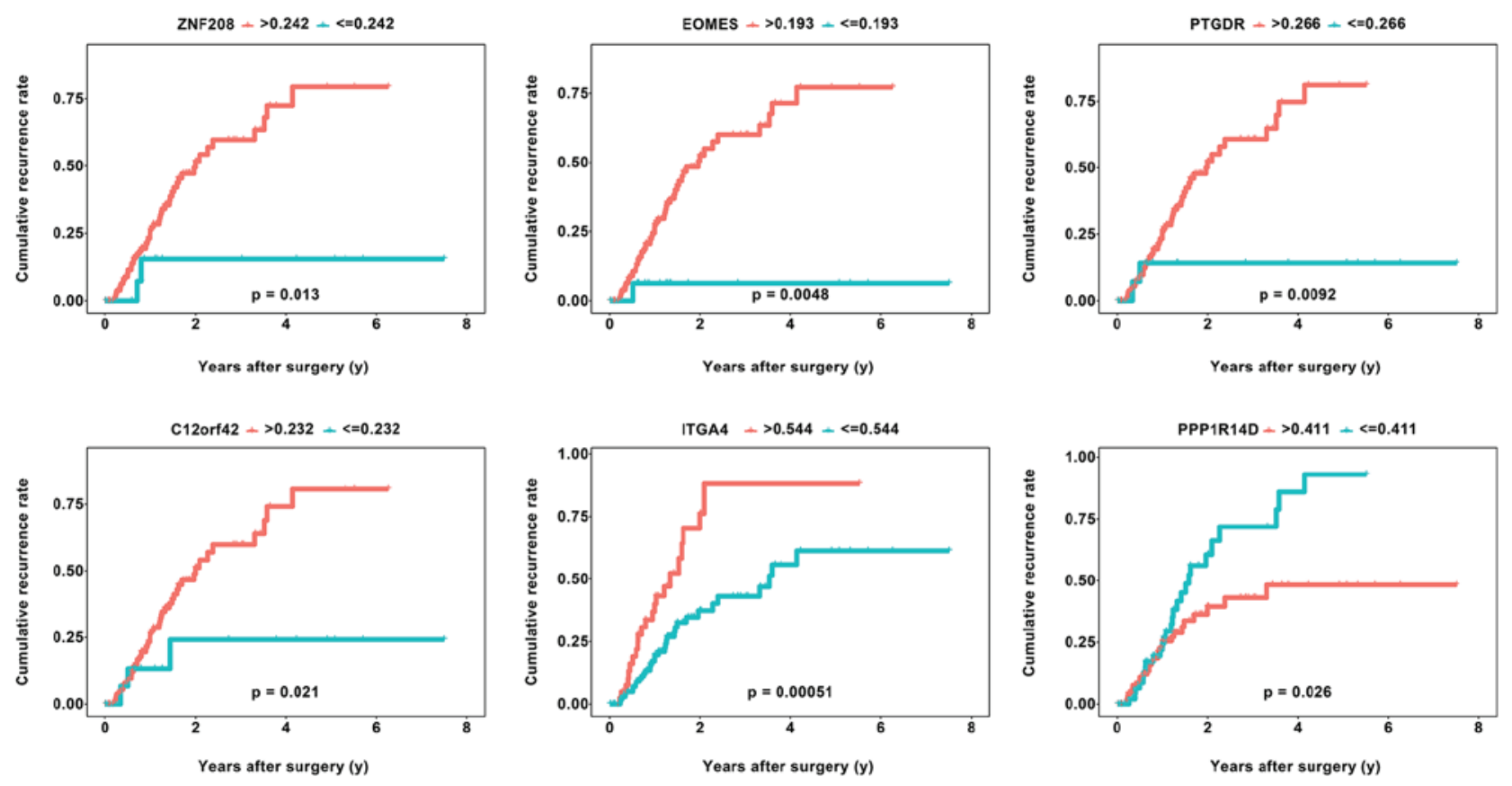

Figure 5. Cumulative recurrence rates associated with 6 DNA methylation-driven genes in pancreatic adenocarcinoma. The cutoff value is displayed above the recurrence curve. The log-rank $\mathrm{P}$ is displayed below the recurrence curve. The $\mathrm{x}$-axis and $\mathrm{y}$-axis display the survival time (years) and cumulative tumor recurrence rate, respectively. ZNF208, zinc finger protein 208; EOMES, eomesodermin; PTGDR, prostaglandin D2 receptor; C12orf42, chromosome 12 open reading frame 42; ITGA4, integrin subunit $\alpha$; PPP1R14D, protein phosphatase 1 regulatory inhibitor subunit 14D.

CI: $1.035-19.345)$ and ITGA4 (P=0.003, HR: $2.244,95 \%$ CI: 1.317-3.822) were still significantly associated with RFS, indicating that PTGDR, EOMES, ZNF208 and ITGA4 were predictors of RFS independent of the TNM stage. However, as the number of patients was small, no significant association was obtained for $\mathrm{C} 12$ orf42 ( $\mathrm{P}=0.056, \mathrm{HR}$ : 3.329, 95\% CI: 0.970-11.424) and PPP1R14D (P=0.076, HR: 0.612, 95\% CI: $0.355-1.053)$, although the results almost reached statistical significance.

Validation of the gene expression of the 7 DNA methylationdriven genes. In the TCGA PAAD cohort (178 PAAD tissues and 4 normal tissues), EOMES $(\mathrm{P}<0.001)$, C12orf42 $(\mathrm{P}<0.001)$, ITGA4 $(\mathrm{P}<0.001)$, DOCK8 $(\mathrm{P}<0.001)$, PTGDR $(\mathrm{P}<0.001)$, ZNF208 $(\mathrm{P}<0.001)$ and PPP1R14D $(\mathrm{P}<0.001)$ were significantly differentially expressed between PAAD tissues and normal tissues. To verify these results, the expression levels of the 7 DNA methylation-driven genes were assessed in the GSE15471 PAAD cohort (35 PAAD tissues and 35 normal tissues). Consistent with the results of the initial analysis, the mean expression levels of EOMES ( $\mathrm{P}=0.024), \mathrm{C} 12$ orf42 $(\mathrm{P}=0.002)$, ITGA4 $(\mathrm{P}<0.001)$ and DOCK8 $(\mathrm{P}<0.001)$ were significantly differentially expressed between PAAD tissues and normal tissues according to the analysis with the limma package in $\mathrm{R}$. However, the differences in expression levels of PTGDR $(\mathrm{P}=0.160), \mathrm{ZNF} 208(\mathrm{P}=0.178)$ and PPP1R14D $(\mathrm{P}=0.222)$ did not reach statistical significance. In addition, the expression of PTGDR, ZNF208 and PPP1R14D was also verified in a larger dataset (GSE28735 PAAD cohort, including 45 PAAD tissues and 45 normal tissues), and the results indicated that PPP1R14D $(\mathrm{P}=0.027)$ and ZNF208 $(\mathrm{P}=0.033)$, but not PTGDR $(\mathrm{P}=0.307)$, were differentially expressed between PAAD tissues and normal tissues.
GSEA. To determine the potential function of the 7 DNA methylation-driven genes, a GSEA was performed to map them using the Encyclopedia of Genes and Genomes (KEGG) pathways database. Under the cut-off criterion of $\mathrm{P}<0.05$, PAAD samples in the high DNA methylation groups for ZNF208, EOMES, PTGDR, C12orf42 and ITGA4 were all most significantly enriched in 'neuroactive ligand receptor interaction' (Fig. 6; Table SV). PAAD samples in the high DNA methylation group for DOCK8 were most significantly enriched in 'basal cell carcinoma' (Fig. 6; Table SV). PAAD samples in the high DNA methylation group for PPP1R14D were most significantly enriched in 'glycolysis gluconeogenesis' (Fig. 6; Table SV).

\section{Discussion}

DNA methylation is closely associated with the occurrence and progression of cancer. In general, there are two different states of abnormal DNA methylation, namely hypomethylation and hypermethylation. It is now widely accepted that abnormal DNA hypermethylation in and around the promoter region results in gene silencing, while hypomethylation results in gene activation. DNA analysis of different tumor cells suggested that the probability of gene mutations in cancer cells was lower than expected (21). However, at the transcriptional level, up to $5 \%$ of known tumor suppressor genes exhibit gene silencing caused by hypermethylation in the promoter region in colorectal cancer, suggesting that abnormal DNA methylation may be more responsible for malignant cell transformation than gene mutations (22).

Compared with normal cells, the methylation levels of tumor cell genomes change substantially; tumor cell genomes are characterized by DNA hypomethylation of the whole genome and 

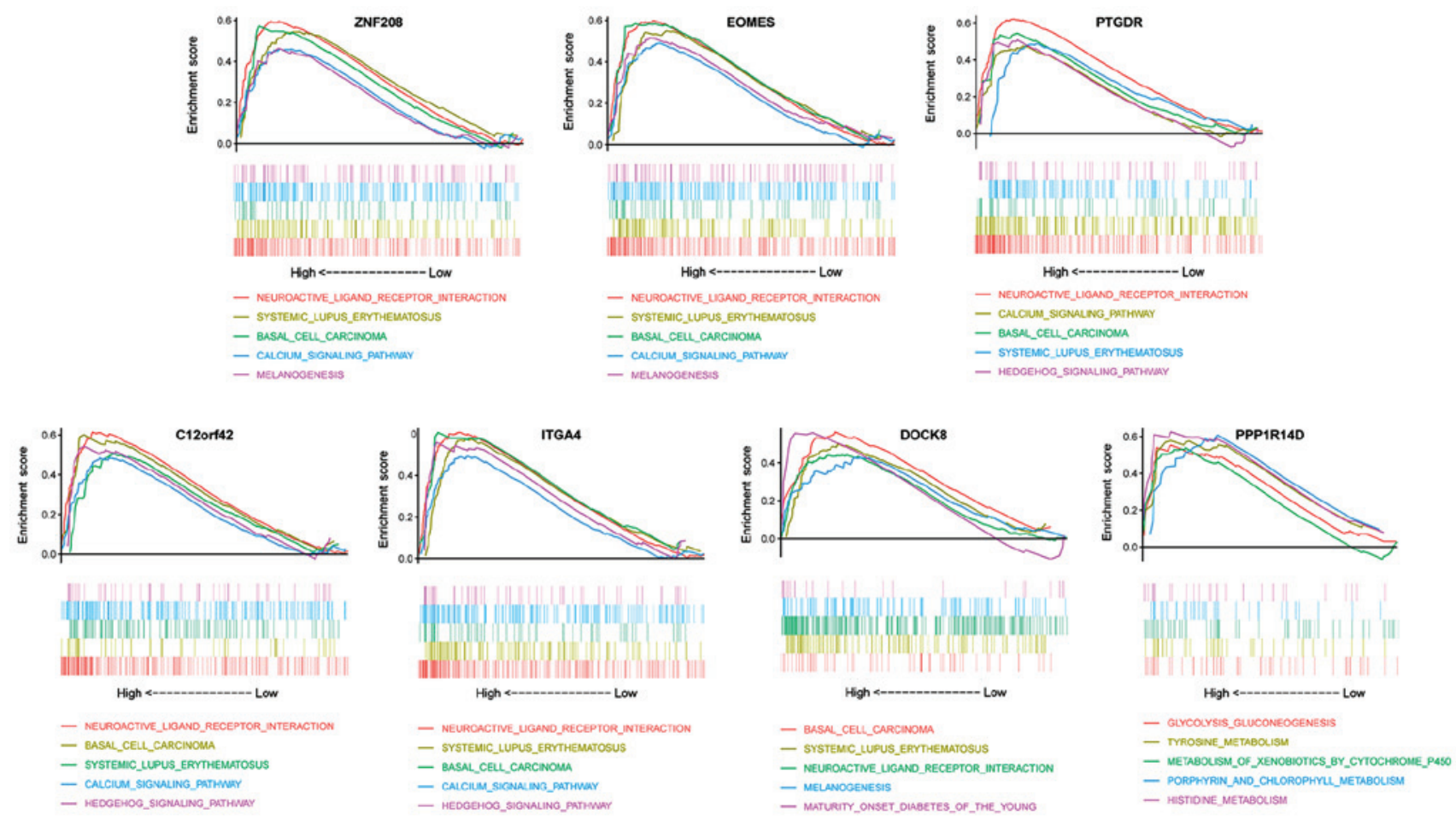

Figure 6. Gene set enrichment analysis of DNA methylation-driven genes. The top five functional gene sets enriched in pancreatic adenocarcinoma samples with high levels of DNA methylation in DNA methylation-driven genes. ZNF208, zinc finger protein 208; EOMES, eomesodermin; PTGDR, prostaglandin D2 receptor; C12orf42, chromosome 12 open reading frame 42; ITGA4, integrin subunit $\alpha$ 4; DOCK8, dedicator of cytokinesis 8; PPP1R14D, protein phosphatase 1 regulatory inhibitor subunit $14 \mathrm{D}$.

abnormal hypermethylation of $\mathrm{CpG}$ islands in local promoter regions. Numerous studies have already been performed regarding the abnormal DNA methylation of the cancer cell genome, particularly the gene silencing of tumor suppressor genes caused by DNA hypermethylation in pancreatic cancer (23-27). Studies have confirmed that DNA hypermethylation is closely linked to PAAD cells evading apoptosis; obtaining sustained proliferation signals; exhibiting insensitivity to growth inhibition signals, tissue infiltration and metastasis; and obtaining infinite replication potential (23-27). The infinite replication potential of cancer cells is mainly linked to increased telomerase activity. Telomerase solves the problem of terminal end deletion during DNA replication at the transcriptional level (28). Normally, telomerase is active only in fertilized eggs and stem cells, while various types of tumor cell have highly active telomerase (28). In the present study, 6 DNA methylation-driven genes (ZNF208, EOMES, PTGDR, C12orf42, ITGA4 and DOCK8) were hypermethylated. ZNF208 has been indicated to be associated with leukocyte telomere length (LTL) (29). LTL-associated genes affect the cancer risk in adults and increased telomere length is associated with an elevated risk of glioma, melanoma and lung cancer in adults (30-33). In addition, downregulation of the mRNA levels of ZNF208, a key tumor suppressor gene in gastric cancer, may contribute to the extensive dissemination of cancer cells in the abdominal cavity (34). The present study indicated that ZNF208 is hypermethylated and has low mRNA expression levels in PAAD compared to normal tissues, which may promote the occurrence and progression of PAAD.

DNA hypomethylation includes DNA hypomethylation of specific proto-oncogenes and DNA hypomethylation of the whole genome. Recent studies have demonstrated that abnormal hypomethylation of specific proto-oncogenes is also critical for the progression and development of pancreatic cancer (35). In the present study, PPP1R14D was hypomethylated and had a relatively low mRNA expression in PAAD. In a previous study, PPP1R14D, a metabolic signaling protein, was indicated to be differentially expressed between normal and pathological conditions of the brain associated with diabetes (36). Diabetes is considered to be a crucial risk factor for PAAD (37). Therefore, PPP1R14D may be involved in the development of PAAD.

Although numerous methods have been developed for the diagnosis of PAAD, each method has its own strengths and weaknesses, and no single method is absolutely superior to the others. The method of choice depends on the resolution and genome coverage requirements, and these two parameters dictate the experimental cost (38). For instance, sequencing approaches have the advantage of providing quantitative information with regard to the methylation state of each $\mathrm{CpG}$ and allowing for repeated analysis of methylation and rare methylation variants, which cannot be performed via microarrays. Furthermore, it is possible to apply sequencing approaches to analyze the DNA methylation of regions without previous sequence information. However, despite the quickly decreasing cost of large-scale sequencing technologies, these technologies have certain major weaknesses, including library bias, high cost and low availability, as well as data management and analysis difficulties. In addition, methods based on bisulfite conversion and sequencing require a large number of bioinformatics resources for calling bases, aligning sequence and performing the statistical analysis. CpG-specific array technology, including DNA methylation arrays, are alternative options that may be used to determine a genome-wide DNA methylation profile. In comparison to 
sequencing technologies, DNA methylation arrays are less expensive and allow for profiling of a large number of samples, although the resolution is reduced. DNA methylation assays take less time and are less labor-intensive (39-41).

Advances in the technologies used to detect genome-wide epigenetic alterations greatly assist us in developing biomarkers for early-stage tumor diagnosis (42). DNA methylation maintains the genomic structure while controlling gene expression. Aberrant DNA methylation usually occurs in promoter regions of the transcription factors that are involved in the development and proliferative process of cancers (43). For instance, hypomethylation of the mesothelin promoter causes mesothelioma (44), and hypomethylated Alu and long interspersed element-1 retrotransposons may lead to lung cancer (45). However, peritoneal mesothelioma has been indicated to feature DNA hypermethylation (46). According to all of these data, DNA methylation is able to perfectly represent and reflect the molecular changes in human tumors in the early stage. Therefore, it may be a useful biomarker facilitating the diagnosis of malignant tumors in the early stage. The present study focused on confirming the validity of highly sensitive biomarkers that may be even more sensitive when used in combination with other biomarkers for the detection of malignancies.

Epigenetic changes due to DNA methylation are one of the important features of the progression of cells to malignancy. Therefore, changes in DNA methylation may be an important facilitator of the early diagnosis of cancer (47). Japanese scholars have collected the fluid from normal pancreases and those with cancer and chronic pancreatitis and used quantitative methylation-specific PCR to detect the degree of methylation of 6 genes, including p16 and cyclin D2 (47). Experiments have revealed that, compared with other methods, the measurement of abnormal methylation of specific genes in the pancreatic fluid is able to more accurately identify pancreatic lesions, leading to the effective early detection of pancreatic cancer (47). In the present study, the AUCs for distinguishing between normal pancreatic tissue and PAAD for the 7 DNA methylation-driven genes, ZNF208, EOMES, PTGDR, C12orf42, ITGA4, DOCK8 and PPP1R14D, were $>0.8$, indicating that the 7 DNA methylation-driven genes exhibited excellent diagnostic efficiency for PAAD.

Unlike genetic mutations, epigenetic changes are mostly reversible. Therefore, targeting epigenetic changes in cells using methylation-associated drugs to alter the methylation status may become a novel treatment for malignant tumors. At present, there are mainly two types of reversal method: One is the use of an antisense oligonucleotide, which is able to inhibit DNA methyltransferase activity, leading to expression of the methylated gene, and the second is the use of a cytidine analog that may covalently bind to DNA methylase, reducing the biological activity of the enzyme, thereby activating genes that are inactivated by methylation (48). At present, the more commonly used cytidine analogs include 5-azacytidine and decitabine. Studies on different pancreatic cancer cell lines cultured in vitro confirmed that 5-azacytidine changes the methylation status of the tumor cell genome through demethylation, thereby inhibiting tumor cell proliferation and promoting apoptosis (48). In clinical trials, studies on myelodysplastic syndromes and acute myeloid leukemia have indicated that 5-azacytidine is able to increase complete response rates by $10-17 \%$ and prolong patient survival $(49,50)$. In the present study, 6 of the 7 DNA methylation-driven genes identified were significantly associated with
OS and the RFS. Five DNA methylation-driven genes (ZNF208, EOMES, PTGDR, C12orf42 and ITGA4) were significantly negatively associated with the OS time and positively associated with the recurrence rate. PPP1R14D was significantly positively associated with the OS time and negatively associated with the recurrence rate. These genes are likely to become novel molecular targets in pancreatic cancer treatments aimed at correcting abnormal DNA methylation to prevent or even reverse cell cancerization.

The 7 DNA methylation-driven genes (ZNF208, EOMES, PTGDR, C12orf42, ITGA4, DOCK8 and PPP1R14D) identified in the present study were previously reported to be associated with other cancer types. ZNF208 acts as a family member of ZNF proteins that contain Kruppel-associated box domains, which may participate in transcriptional regulation (51). In a study by Hirbe et al (51), immunohistochemistry helped detect ZNF208 protein expression in metastatic tumors. In addition, very large genome-wide association studies (GWAS) have identified an association between ZNF208 and interindividual variation in LTL (52). A recently performed Mendelian randomization study demonstrated the impact of single nucleotide polymorphisms (SNPs) associated with LTL on adult cancer risk and further indicated that the genetic predisposition to increased telomere length may increase the risk of lung cancer, melanoma and glioma in adults (52).

The expression of EOMES has been reported to be negatively associated with tumor-infiltrating lymphocyte (TIL) function in non-small cell lung cancers (NSCLCs) in the early stage, suggesting that the functionality of TILs associated with early-stage NSCLCs may be influenced by an exhaustion program marked by EOMES expression (53). Chang et al (54) indicated that PTGDR was hypermethylated in endometrial cancer and ovarian cancer tissues. Przybylski et al (55) identified a novel type of chromosomal translocation, t(12;14)(q23; q11.2), in T-lymphoblastic lymphoma between T-cell receptor delta-deleting elements ( $\mathrm{T}$-cell receptor delta recombining element and $\mathrm{T}$ cell receptor alpha joining 61) and the hypothetical gene $\mathrm{C} 12$ orf42. In a recently performed melanoma GWAS meta-analysis (involving 12,874 cases and 23,203 controls), SNPs near DOCK8 reached global significance (56). Morandi et al (57) reported that ITGA4 was hypermethylated in oral squamous cell carcinoma but not in samples from normal healthy donors. With regard to PPP1R14D, the Pearson correlation between the $\mathrm{CpG}$ sites with different methylation levels and the gene expression values in NSCLCs in the early stage reached statistical significance (58).

As reported by previous studies, RASSF1A, p16, SOCS-1 and NPTX2 are abnormally methylated in pancreatic cancer and are critical for the pathogenesis of this cancer type (12-14). However, in the present study, neither RASSF1A nor p16 was present in the TCGA database; thus, they were not included in the prognostic analysis. As better sequencing approaches emerge in the future, prognostic analyses will improve. In the present study, to identify the most significant genes associated with PAAD, thresholds for the screening of DEGs were set at an $\mathrm{FDR}<0.05$ and $\log _{2} \mathrm{FCl}>2$; hence, genes in the prognostic analysis may exhibit more obvious statistical and biological significance. However, SOCS-1 and NPTX2 did not reach the thresholds of $\mathrm{FDR}<0.05$ and $\log _{2} \mathrm{FCl}>2$. 
Of note, several limitations of the present study should be considered. First, the ethnicities of the population in the TCGA database, which is from the US, are primarily Latino and Caucasian, and it is necessary to substantiate the extrapolation of the results of the present study to other ethnic groups. Second, the prognostic analyses of these 7 DNA methylation-driven genes were based on a retrospective study, so the use of these genes as biomarkers requires prospective multicenter validation. Third, further studies are required to investigate and validate the functions and molecular mechanisms of the pathogenesis and progression of PAAD with regard to these 7 DNA methylation-driven genes in in vivo and in vitro experiments.

In summary, 7 DNA methylation-driven genes were identified by a comprehensive analysis of DNA methylation and mRNA expression data from 188 clinical samples. The 7 DNA methylation-driven genes will contribute to the understanding of how pancreatic cancer occurs and develops, as well as provide novel ways to diagnose and treat pancreatic cancer.

\section{Acknowledgements}

The authors would like to thank Dr Shijun Wei from the Department of Medical Genetics, Shandong University School of Medicine, Jinan, Shandong, China for providing technical support.

\section{Funding}

The design of the current study and the collection, analysis and interpretation of data in this work were supported by the Basic Research Fund of the Ocean University of China (grant no. 201562018).

\section{Availability of data and materials}

All data generated or analyzed during the present study are included in this published article.

\section{Authors' contributions}

$\mathrm{XT}$ and $\mathrm{WZ}$ were responsible for the conception and design of this research. YY, PL, TW and XC were responsible for data collection, collation and analysis. WZ and SS were responsible for data analysis and interpretation, and manuscript content. $\mathrm{XT}$ was responsible for approving the publication of the final version. XT agreed to be accountable for all aspects of the work in ensuring that questions related to the accuracy or integrity of any part of the work are appropriately investigated and resolved.

\section{Ethics approval and consent to participate}

Not applicable.

\section{Patient consent for publication}

Not applicable.

\section{Competing interests}

The authors declare that they have no competing interests.

\section{References}

1. Rahib L, Smith BD, Aizenberg R, Rosenzweig AB, Fleshman JM and Matrisian LM: Projecting cancer incidence and deaths to 2030: The unexpected burden of thyroid, liver, and pancreas cancers in the United States. Cancer Res 74: 2913-2921, 2014.

2. Higuera O, Ghanem I, Nasimi R, Prieto I, Koren L and Feliu J: Management of pancreatic cancer in the elderly. World $\mathrm{J}$ Gastroenterol 22: 764-775, 2016.

3. Crippa S, Capurso G, Camma C, Fave GD, Castillo CF and Falconi M: Risk of pancreatic malignancy and mortality in branch-duct IPMNs undergoing surveillance: A systematic review and meta-analysis. Dig Liver Dis 48: 473-479, 2016.

4. Chiaravalli M, Reni M and O'Reilly EM: Pancreatic ductal adenocarcinoma: State-of-the-art 2017 and new therapeutic strategies. Cancer Treat Rev 60: 32-43, 2017.

5. Kota J, Hancock J, Kwon J and Korc M: Pancreatic cancer: Stroma and its current and emerging targeted therapies. Cancer Lett 391: 38-49, 2017.

6. Giovannetti E, van der Borden CL, Frampton AE, Ali A, Firuzi O and Peters GJ: Never let it go: Stopping key mechanisms underlying metastasis to fight pancreatic cancer. Semin Cancer Biol 44: 43-59, 2017.

7. Zhang ML, Lu S, Zhou L and Zheng SS: Correlation between ECT2 gene expression and methylation change of ECT2 promoter region in pancreatic cancer. Hepatobiliary Pancreat Dis Int 7: 533-538, 2008.

8. Jones PA: Functions of DNA methylation: Islands, start sites, gene bodies and beyond. Nat Rev Genet 13: 484-492, 2012.

9. Zhang M, Lv X, Jiang Y, Li G and Qiao Q: Identification of aberrantly methylated differentially expressed genes in glioblastoma multiforme and their association with patient survival. Exp Ther Med 18: 2140-2152, 2019.

10. Pan R, Zhou C, Dai J, Ying X, Yu H, Zhong J, Zhang Y, Wu B, Mao Y, Wu D, et al: Endothelial PAS domain protein 1 gene hypomethylation is associated with colorectal cancer in Han Chinese. Exp Ther Med 16: 4983-4990, 2018.

11. Kisiel JB, Raimondo M, Taylor WR, Yab TC, Mahoney DW, Sun Z, Middha S, Baheti S, Zou H, Smyrk TC, et al: New DNA methylation markers for pancreatic cancer: Discovery, tissue validation, and pilot testing in pancreatic juice. Clin Cancer Res 21: 4473-4481, 2015.

12. Pan FP, Zhou HK, Bu HQ, Chen ZQ, Zhang H, Xu LP, Tang J, Yu QJ, Chu YQ, Pan J, et al: Emodin enhances the demethylation by 5 -Aza-CdR of pancreatic cancer cell tumor-suppressor genes P16, RASSF1A and ppENK. Oncol Rep 35: 1941-1949, 2016.

13. Komazaki T, Nagai H, Emi M, Terada Y, Yabe A, Jin E, Kawanami O, Konishi N, Moriyama Y, Naka T and Kishimoto T: Hypermethylation-associated inactivation of the SOCS-1 gene, a JAK/STAT inhibitor, in human pancreatic cancers. Jpn J Clin Oncol 34: 191-194, 2004.

14. Zhang L, Gao J, Li Z and Gong Y: Neuronal pentraxin II (NPTX2) is frequently down-regulated by promoter hypermethylation in pancreatic cancers. Dig Dis Sci 57: 2608-2614, 2012.

15. Integrated Genomic Characterization of Pancreatic Ductal Adenocarcinoma. Cancer Cell 32: 185-203.e113, 2017.

16. Cedoz PL, Prunello M, Brennan K and Gevaert O: MethylMix 2.0: An R package for identifying DNA methylation genes. Bioinformatics 34: 3044-3046, 2018.

17. Cleveland P, Gill KR, Coe SG, Woodward TA, Raimondo M, Jamil L, Gross SA, Heckman MG, Crook JE and Wallace MB: An evaluation of risk factors for inadequate cytology in EUS-guided FNA of pancreatic tumors and lymph nodes. Gastrointest Endosc 71: 1194-1199, 2010.

18. Li L, Li C, Mao H, Du Z, Chan WY, Murray P, Luo B, Chan AT, Mok TS, Chan FK, et al: Epigenetic inactivation of the $\mathrm{CpG}$ demethylase TET1 as a DNA methylation feedback loop in human cancers. Sci Rep 6: 26591, 2016.

19. Reis AH, Vargas FR and Lemos B: Biomarkers of genome instability and cancer epigenetics. Tumour Biol 37: 13029-13038, 2016.

20. Robinson MD, McCarthy DJ and Smyth GK: edgeR: A Bioconductor package for differential expression analysis of digital gene expression data. Bioinformatics 26: 139-140, 2010.

21. Thomas RK, Baker AC, Debiasi RM, Winckler W, Laframboise T, Lin WM, Wang M, Feng W, Zander T, MacConaill L, et al: High-throughput oncogene mutation profiling in human cancer. Nat Genet 39: 347-351, 2007.

22. Schuebel KE, Chen W, Cope L, Glöckner SC, Suzuki H, Yi JM, Chan TA, Van Neste L, Van Criekinge W, van den Bosch S, et al: Comparing the DNA hypermethylome with gene mutations in human colorectal cancer. PLoS Genet 3: 1709-1723, 2007. 
23. Dammann R, Schagdarsurengin U, Liu L, Otto N, Gimm O, Dralle H, Boehm BO, Pfeifer GP and Hoang-Vu C: Frequent RASSF1A promoter hypermethylation and K-ras mutations in pancreatic carcinoma. Oncogene 22: 3806-3812, 2003.

24. Sato N, Fukushima N, Maitra A, Matsubayashi H, Yeo CJ, Cameron JL, Hruban RH and Goggins M: Discovery of novel targets for aberrant methylation in pancreatic carcinoma using high-throughput microarrays. Cancer Res 63: 3735-3742, 2003.

25. Ueki T, Walter KM, Skinner H, Jaffee E, Hruban RH and Goggins M: Aberrant $\mathrm{CpG}$ island methylation in cancer cell lines arises in the primary cancers from which they were derived. Oncogene 21: 2114-2117, 2002.

26. Fukushima N, Sato N, Ueki T, Rosty C, Walter KM, Wilentz RE, Yeo CJ, Hruban RH and Goggins M: Aberrant methylation of preproenkephalin and p16 genes in pancreatic intraepithelial neoplasia and pancreatic ductal adenocarcinoma. Am J Pathol 160: 1573-1581, 2002.

27. Kumari A, Srinivasan R and Wig JD: Effect of c-MYC and E2F1 gene silencing and of 5-azacytidine treatment on telomerase activity in pancreatic cancer-derived cell lines. Pancreatology 9: 360-368, 2009

28. Nakamura TM, Morin GB, Chapman KB, Weinrich SL, Andrews WH, Lingner J, Harley CB and Cech TR: Telomerase catalytic subunit homologs from fission yeast and human. Science 277: 955-959, 1997.

29. Ojha J, Codd V, Nelson CP, Samani NJ, Smirnov IV, Madsen NR, Hansen HM, de Smith AJ, Bracci PM, Wiencke JK, et al: Genetic variation associated with longer telomere length increases risk of chronic lymphocytic leukemia. Cancer Epidemiol Biomarkers Prev 25: 1043-1049, 2016.

30. Codd V, Nelson CP, Albrecht E, Mangino M, Deelen J, Buxton JL, Hottenga JJ, Fischer K, Esko T, Surakka I, et al: Identification of seven loci affecting mean telomere length and their association with disease. Nat Genet 45: 422-427, 427e1-2, 2013.

31. Walsh KM, Codd V, Rice T, Nelson CP, Smirnov IV, McCoy LS, Hansen HM, Elhauge E, Ojha J, Francis SS, et al: Longer genotypically-estimated leukocyte telomere length is associated with increased adult glioma risk. Oncotarget 6: 42468-42477, 2015.

32. Iles MM, Bishop DT, Taylor JC, Hayward NK, Brossard M, Cust AE, Dunning AM, Lee JE, Moses EK, Akslen LA, et al: The effect on melanoma risk of genes previously associated with telomere length. J Natl Cancer Inst 106: pii: dju267, 2014.

33. Zhang C, Doherty JA, Burgess S, Hung RJ, Lindström S, Kraft P, Gong J, Amos CI, Sellers TA, Monteiro AN, et al: Genetic determinants of telomere length and risk of common cancers: A Mendelian randomization study. Hum Mol Genet 24: 5356-5366, 2015.

34. Zhang J, Huang JY, Chen YN, Yuan F, Zhang H, Yan FH, Wang MJ, Wang G, Su M, Lu G, et al: Whole genome and transcriptome sequencing of matched primary and peritoneal metastatic gastric carcinoma. Sci Rep 5: 13750, 2015.

35. Fernandez-Zapico ME, Gonzalez-Paz NC, Weiss E, Savoy DN, Molina JR, Fonseca R, Smyrk TC, Chari ST, Urrutia R and Billadeau DD: Ectopic expression of VAV1 reveals an unexpected role in pancreatic cancer tumorigenesis. Cancer Cell 7: 39-49, 2005 .

36. Karthik D and Ravikumar S: Characterization of the brain proteome of rats with diabetes mellitus through two-dimensional electrophoresis and mass spectrometry. Brain Res 1371: 171-179, 2011.

37. Rahn S, Zimmermann V, Viol F, Knaack H, Stemmer K, Peters L, Lenk L, Ungefroren H, Saur D, Schäfer H and Helm O: Diabetes as risk factor for pancreatic cancer: Hyperglycemia promotes epithelial-mesenchymal-transition and stem cell properties in pancreatic ductal epithelial cells. Cancer Lett 415: 129-150, 2018.

38. Jankowska AM, Millward CL and Caldwell CW: The potential of DNA modifications as biomarkers and therapeutic targets in oncology. Expert Rev Mol Diagn 15: 1325-1337, 2015.

39. Heyn $\mathrm{H}$ and Esteller M: DNA methylation profiling in the clinic: Applications and challenges. Nat Rev Genet 13: 679-692, 2012.

40. Olkhov-Mitsel E and Bapat B: Strategies for discovery and validation of methylated and hydroxymethylated DNA biomarkers. Cancer Med 1: 237-260, 2012

41. Rivera CM and Ren B: Mapping human epigenomes. Cell 155: 39-55, 2013.

42. Bird A: DNA methylation patterns and epigenetic memory. Genes Dev 16: 6-21, 2002

43. Razin A and Riggs AD: DNA methylation and gene function. Science 210: 604-610, 1980
44. Tan K, Kajino K, Momose S, Masaoka A, Sasahara K, Shiomi K, Izumi H, Abe M, Ohtsuji N, Wang T, et al: Mesothelin (MSLN) promoter is hypomethylated in malignant mesothelioma, but its expression is not associated with methylation status of the promoter. Hum Pathol 41: 1330-1338, 2010.

45. Daskalos A, Nikolaidis G, Xinarianos G, Savvari P, Cassidy A, Zakopoulou R, Kotsinas A, Gorgoulis V, Field JK and Liloglou T: Hypomethylation of retrotransposable elements correlates with genomic instability in non-small cell lung cancer. Int J Cancer 124: 81-87, 2009.

46. Hama R, Watanabe Y, Shinada K, Yamada Y, Ogata Y, Yoshida Y, Tamura T, Hiraishi T, Oikawa R, Sakurai J, et al: Characterization of DNA hypermethylation in two cases of peritoneal mesothelioma. Tumour Biol 33: 2031-2040, 2012.

47. Matsubayashi H, Canto M, Sato N, Klein A, Abe T, Yamashita K, Yeo CJ, Kalloo A, Hruban R and Goggins M: DNA methylation alterations in the pancreatic juice of patients with suspected pancreatic disease. Cancer Res 66: 1208-1217, 2006.

48. Missiaglia E, Donadelli M, Palmieri M, Crnogorac-Jurcevic T, Scarpa A and Lemoine NR: Growth delay of human pancreatic cancer cells by methylase inhibitor 5-aza-2'-deoxycytidine treatment is associated with activation of the interferon signalling pathway. Oncogene 24: 199-211, 2005.

49. Fenaux P, Mufti GJ, Hellstrom-Lindberg E, Santini V, Finelli C, Giagounidis A, Schoch R, Gattermann N, Sanz G, List A, et al: Efficacy of azacitidine compared with that of conventional care regimens in the treatment of higher-risk myelodysplastic syndromes: A randomised, open-label, phase III study. Lancet Oncol 10: 223-232, 2009.

50. Silverman LR, McKenzie DR, Peterson BL, Holland JF, Backstrom JT, Beach CL and Larson RA; Cancer and Leukemia Group B: Further analysis of trials with azacitidine in patients with myelodysplastic syndrome: Studies 8421,8921 , and 9221 by the Cancer and Leukemia Group B. J Clin Oncol 24: 3895-3903, 2006.

51. Hirbe AC, Dahiya S, Miller CA, Li T, Fulton RS, Zhang X, McDonald S, DeSchryver K, Duncavage EJ, Walrath J, et al: Whole exome sequencing reveals the order of genetic changes during malignant transformation and metastasis in a single patient with NF1-plexiform neurofibroma. Clin Cancer Res 21: 4201-4211, 2015.

52. Walsh KM, Whitehead TP, de Smith AJ, Smirnov IV, Park M, Endicott AA, Francis SS, Codd V, ENGAGE Consortium Telomere Group, Samani NJ, et al: Common genetic variants associated with telomere length confer risk for neuroblastoma and other childhood cancers. Carcinogenesis 37: 576-582, 2016.

53. O'Brien SM, Klampatsa A, Thompson JC, MartinezMC,Hwang WT, Rao AS, Standalick JE, Kim S, Cantu E, Litzky LA, et al: Function of human tumor-infiltrating lymphocytes in early-stage non-small cell lung cancer. Cancer Immunol Res 7: 896-909, 2019.

54. Chang CC, Wang HC, Liao YP, Chen YC, Weng YC, Yu MH and Lai HC: The feasibility of detecting endometrial and ovarian cancer using DNA methylation biomarkers in cervical scrapings. J Gynecol Oncol 29: e17, 2018.

55. Przybylski GK, Dittmann K, Grabarczyk P, Dölken G, Gesk S, Harder L, Landmann E, Siebert R and Schmidt CA: Molecular characterization of a novel chromosomal translocation $\mathrm{t}(12 ; 14)$ (q23;q11.2) in T-lymphoblastic lymphoma between the T-cell receptor delta-deleting elements (TRDREC and TRAJ61) and the hypothetical gene C12orf42. Eur J Haematol 85: 452-456, 2010.

56. Duffy DL, Zhu G, Li X, Sanna M, Iles MM, Jacobs LC, Evans DM, Yazar S, Beesley J, Law MH, et al: Novel pleiotropic risk loci for melanoma and nevus density implicate multiple biological pathways. Nat Commun 9: 4774, 2018.

57. Morandi L, Gissi D, Tarsitano A, Asioli S, Gabusi A, Marchetti C, Montebugnoli L and Foschini MP: CpG location and methylation level are crucial factors for the early detection of oral squamous cell carcinoma in brushing samples using bisulfite sequencing of a 13-gene panel. Clin Epigenetics 9: 85, 2017.

58. Lokk K, Vooder T, Kolde R, Välk K, Võsa U, Roosipuu R, Milani L, Fischer K, Koltsina M, Urgard E, et al: Methylation markers of early-stage non-small cell lung cancer. PLoS One 7: e39813, 2012

This work is licensed under a Creative Commons Attribution-NonCommercial-NoDerivatives 4.0 International (CC BY-NC-ND 4.0) License. 